\title{
ACCELERATION, CONTAINMENT, AND EMISSION OF VERY LOW ENERGY SOLAR FLARE PARTICLES
}

\author{
R. P. LIN, R. E. McGUIRE*, and K. A. ANDERSON* \\ Space Sciences Laboratory, University of California, Berkeley, Calif. 94720, U.S.A.
}

\begin{abstract}
We present the first observations of protons down to $44 \mathrm{keV}$ and electrons down to $\$ 2 \mathrm{keV}$ emitted in an impulsive solar particle event. The observations are from the Apollo 15 Subsatellite during a flare event which began on September 1, 1971. We obtain a lower limit estimate of the energy contained in $\gtrsim 0.05 \mathrm{MeV}$ protons in the flare. The effects of adiabatic deceleration in the interplanetary medium and $\mathrm{d} E / \mathrm{d} x$ energy loss in the corona are discussed. We conclude that energetic protons may constitute a major energy release in large solar flares.
\end{abstract}

\section{Introduction}

It has been known for some time that in many small flares (importance 1 or subflares) energetic, $20-100 \mathrm{keV}$ electrons accelerated during the flash phase constitute a major portion of the total flare energy (Lin and Hudson, 1971; Lin, 1973; Kane, 1974). If the electron spectrum extends to energies as low as $\$ 5 \mathrm{keV}$ as indicated by observations, then the electrons would clearly contain the bulk of the flare's energy. Satellites have measured the proton component in large proton flares down to energies of $\sim 0.3 \mathrm{MeV}$. These observations indicate that protons to energies of $0.3 \mathrm{MeV}$ are accelerated and emitted into the interplanetary medium by these flares and that the energy spectrum, which is typically a power law in energy, is still rapidly rising with decreasing energy at $\sim 0.3 \mathrm{MeV}$ (Verzariu and Krimigis, 1972). If the power law proton spectrum extends to even lower energies, then the protons could contain a major portion of the energy in these large flares.

In this paper we present Apollo 15 Subsatellite observations of low energy protons and electrons during the solar flare event of 1 September 1971. These observations cover the range from $\sim 44 \mathrm{keV}$ to $\sim 2 \mathrm{MeV}$ for protons and $\lesssim 2 \mathrm{keV}$ to $\sim 340 \mathrm{keV}$ for electrons. At these low energies the energy change processes of $d E / d x$, adiabatic deceleration and Fermi acceleration play an important role in the propagation of these particles. Here we provide some estimates of the magnitude of energy changes due to these various processes. We also obtain a lower limit to the energy contained in energetic, $>0.04 \mathrm{MeV}$ protons emitted by this flare into the interplanetary medium.

\section{Experimental Details}

The observations reported here are from the University of California particle experiment aboard the Apollo 15 Subsatellite. This Subsatellite was ejected from the Apollo 15 Lunar Science Module on 4 August 1971, into a lunar orbit with initial perilune

* Also Physics Dept., University of California, Berkeley, Calif. 94720, U.S.A. 
of $102 \mathrm{~km}$, apolune of $139 \mathrm{~km}$, and orbit inclination of $28.5^{\circ}$ to the lunar equatorial plane. The Subsatellite samples particle fluxes at $\sim 60 R_{\mathrm{E}}$ distance from the Earth, and during this solar event the Subsatellite was in the distant magnetosheath and magnetotail regions.

The instrumentation in the UCal (Berkeley) experiment consisted of a pair of surface barrier semiconductor telescopes, one of which was open and the other of which was covered by a thin, low $Z$ foil. This foil stopped protons of less than $\sim 340 \mathrm{keV}$ from registering in the telescope. By suitable subtraction of the fluxes observed in the two telescopes, electron and proton fluxes could be identified and measured. Details of this arrangement are given in Anderson et al. (1972).

In addition four large geometric factor, hemispherical plate electrostatic analyzers utilizing channel multiplier detectors provided measurements of electrons at $\sim 0.5$, 2,6 , and $14 \mathrm{keV}$. The $\sim 6$ and $\sim 14 \mathrm{keV}$ detectors were surrounded by plastic anticoincidence jacket to reject penetrating particles and thereby reduce the background. Further details are contained in Anderson et al. (1972).

\section{Observations}

The first major solar particle event observed by the Subsatellite began on 1 September 1971 at $\sim 2000$ UT. The relevant solar, interplanetary and terrestrial times are summarized in Table I. No optical flare was observed but both radio and X-ray emission was reported in the Solar-Geophysical Data Bulletin at $~ 1930$ UT. It is very likely that this event originated in a flare in McMath plage region number 11842 which

TABLE I

Event times

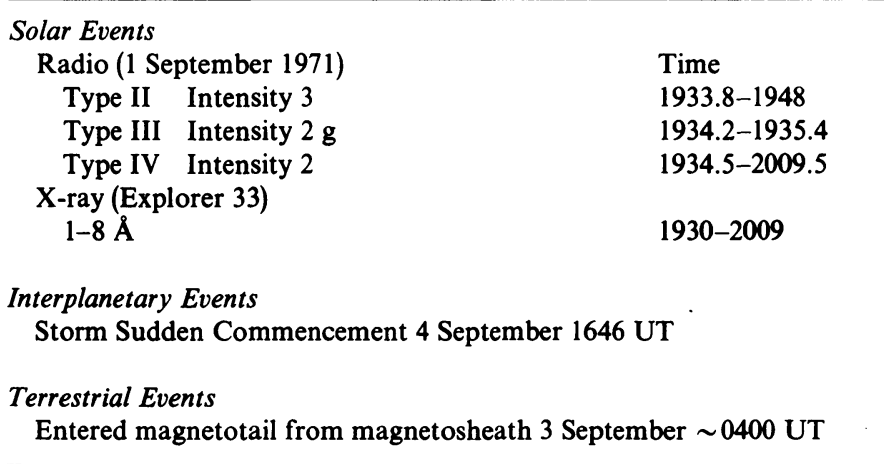

went over the west limb of the Sun August 31. The observations are complicated by several problems.

(a) The onset of this event was not observed by the Subsatellite since it was in the midst of a battery charge. The first particle data was obtained at the beginning of 2 September 1971. 
(b) The Subsatellite was in the magnetosheath and crossed into the magnetotail during the event. It passed on several occasions through the magnetotail plasma sheet which contains large fluxes of protons and electrons in these low energy ranges which would mask the solar particle fluxes.

(c) The gain of the open telescope changed early in the event. Although we do not know the cause of this gain change we were able to estimate its magnitude from a comparison with the foil telescope during electron shadows. The fluxes have been corrected for the gain change.

(d) Due to an engineering oversight the Subsatellite 19 to 8 bit floating point accumulators registers 16-31 counts per accumulation period as 0 to 15 counts per accumulation period.

During the solar event we have attempted to obtain values of the solar particle fluxes which are uncontaminated by these effects. The proton points are plotted in Figure 1. Particularly in the lowest two energy channels it was difficult to separate solar fluxes from terrestrial fluxes, and from background due to penetrating particles. However, the main features of the low energy event are clear from this figure. These are:

(a) There is a distinct velocity dispersion of the peaks down to the $72-130 \mathrm{keV}$ channel with the highest energy peaking earliest.

(b) The profiles are generally similar to those observed previously at higher energies.

(c) There was a storm sudden commencement at $\sim 1700$ UT on 4 September. Associated with this SSC was the characteristic increase in low energy proton fluxes observed in front of an interplanetary shock. These fluxes dominated the intensity-time profile from $\$ 1200$ UT on 4 September. A characteristic flux decrease was observed after the SSC at the beginning of 5 September.

We thus associate the proton fluxes observed in the period $\sim 0000$ UT 2 September to $\sim 1600$ UT 4 September with an impulsive injection of protons at the time of the flare, followed by propagation in the interplanetary medium.

The energy spectrum of the proton event is shown in Figure 2. We have plotted the maximum flux in each energy channel. The spectrum below $\sim 1 \mathrm{MeV}$ is very shallow with $\mathrm{d} J / \mathrm{d} E \propto E^{-0.65}$. Also shown are the $10-30 \mathrm{MeV}$ and $30-60 \mathrm{MeV}$ fluxes from the Solar-Geophysical Data Bulletins. The best fit over the entire spectral range is given by $\mathrm{d} J / \mathrm{d} P=55 \exp (-P / 65)$, where $P$ is particle rigidity in $\mathrm{MeV} / c$.

During this event electrons were observed as well. Their intensity-time profiles are typical of electron events and are shown in Figure 3. Electrons were observed to $\$ 2 \mathrm{keV}$, and the electron energy spectrum, plotted in Figure 4, extends smoothly in a power law $\mathrm{d} J / \mathrm{d} E \propto E^{-1.9}$ to $\lesssim 2 \mathrm{keV}$.

\section{Discussion}

In a preliminary analysis we have estimated the diffusion coefficients for each proton energy channel by comparison with the analytic solution of the diffusion-convection- 


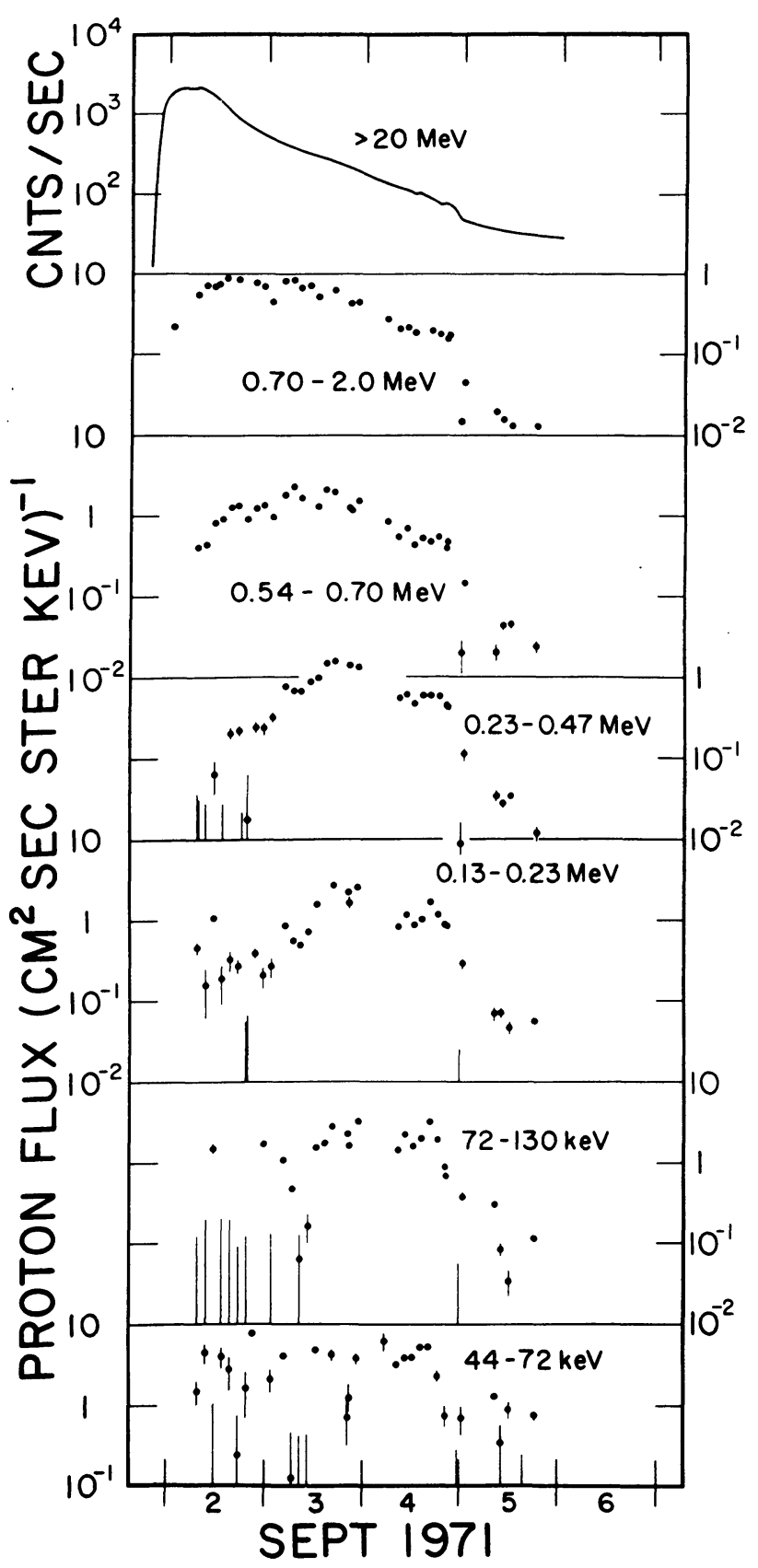

Fig. 1. The low energy proton observations for the fiare event of 1 September 1971. The $>20 \mathrm{MeV}$ profile is from the UCal IMP-6 experiment. All the rest of the data is from the Apollo 15 Subsatellite, chosen at times when the fluxes are free from terrestrial fluxes. Early in the event the 44-72 keV and the $72-130 \mathrm{keV}$ channels may be contaminated. 
energy loss transport equation (Parker, 1965)

$$
\frac{\partial U}{\partial t}+\frac{1}{r^{2}} \frac{\partial}{\partial r}\left(r^{2} V U-r^{2} k \frac{\partial U}{\partial r}\right)-\frac{2 V}{3 r} \frac{\partial}{\partial T}(\alpha T U)=0
$$

$U(r, T, t)$ is the differential number density, $T$ is the kinetic energy, $V$ is the solar wind velocity, assumed constant, and $\alpha \approx 2$ for non-relativistic particles; $r$ and $t$ are distance and time respectively. This has been solved for an impulsive injection of particles at

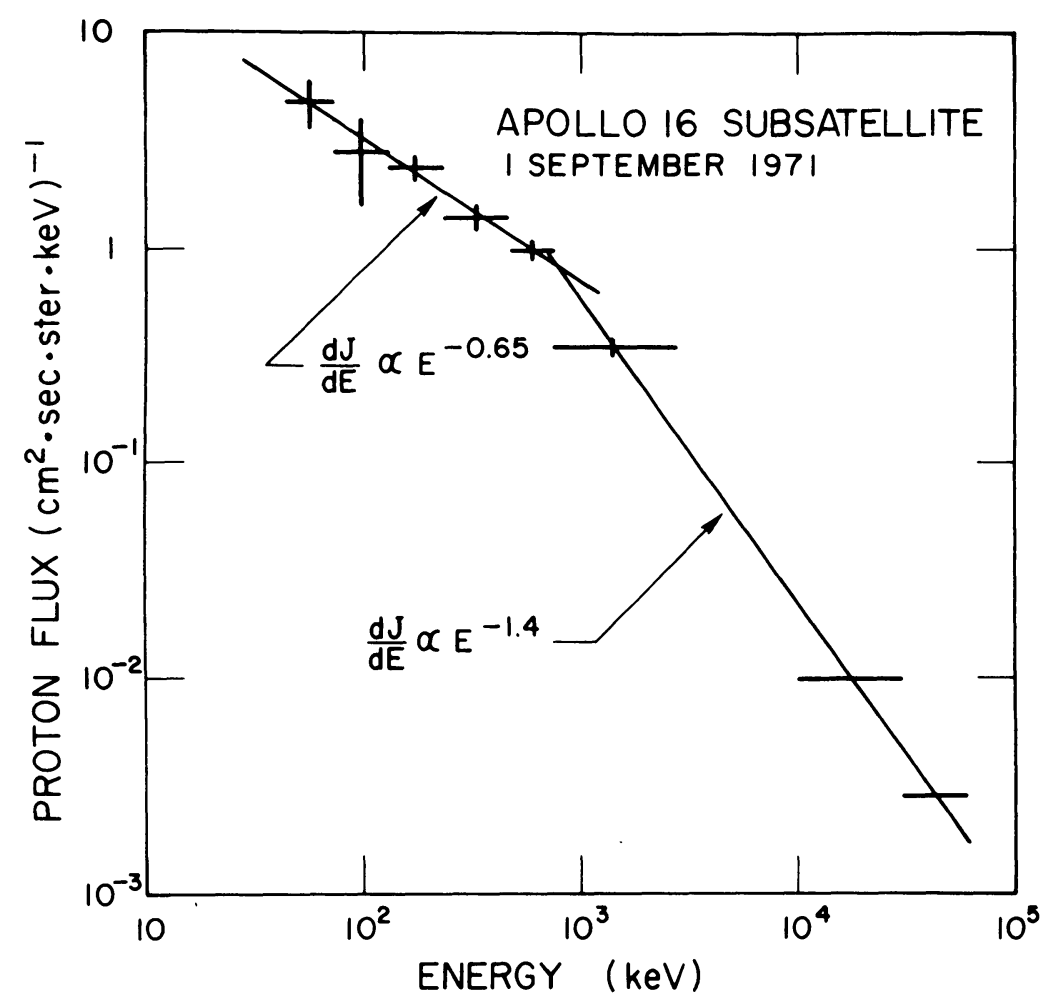

Fig. 2. The proton energy spectrum for the 1 September 1971 flare event, plotted from the maximum flux in each energy interval.

the Sun with $k=k_{0} r$ and with energy dependence $U \propto T^{-\mu}$ (Fisk and Axford, 1968). $\mathrm{Ng}$ and Gleeson (1971) find that for times, $t$, such that

$$
\eta k_{0} t \gg 2\left(r r_{0}\right)^{1 / 2}
$$

where

$$
\begin{aligned}
\eta & =\left[\left(2+V / k_{0}\right)^{2}+16 V(\mu-1) / 3 k_{0}\right]^{1 / 2}, \text { and } \\
r_{0} & =\text { radius of injection surface } \\
r & =\text { radius at point of observation; }
\end{aligned}
$$




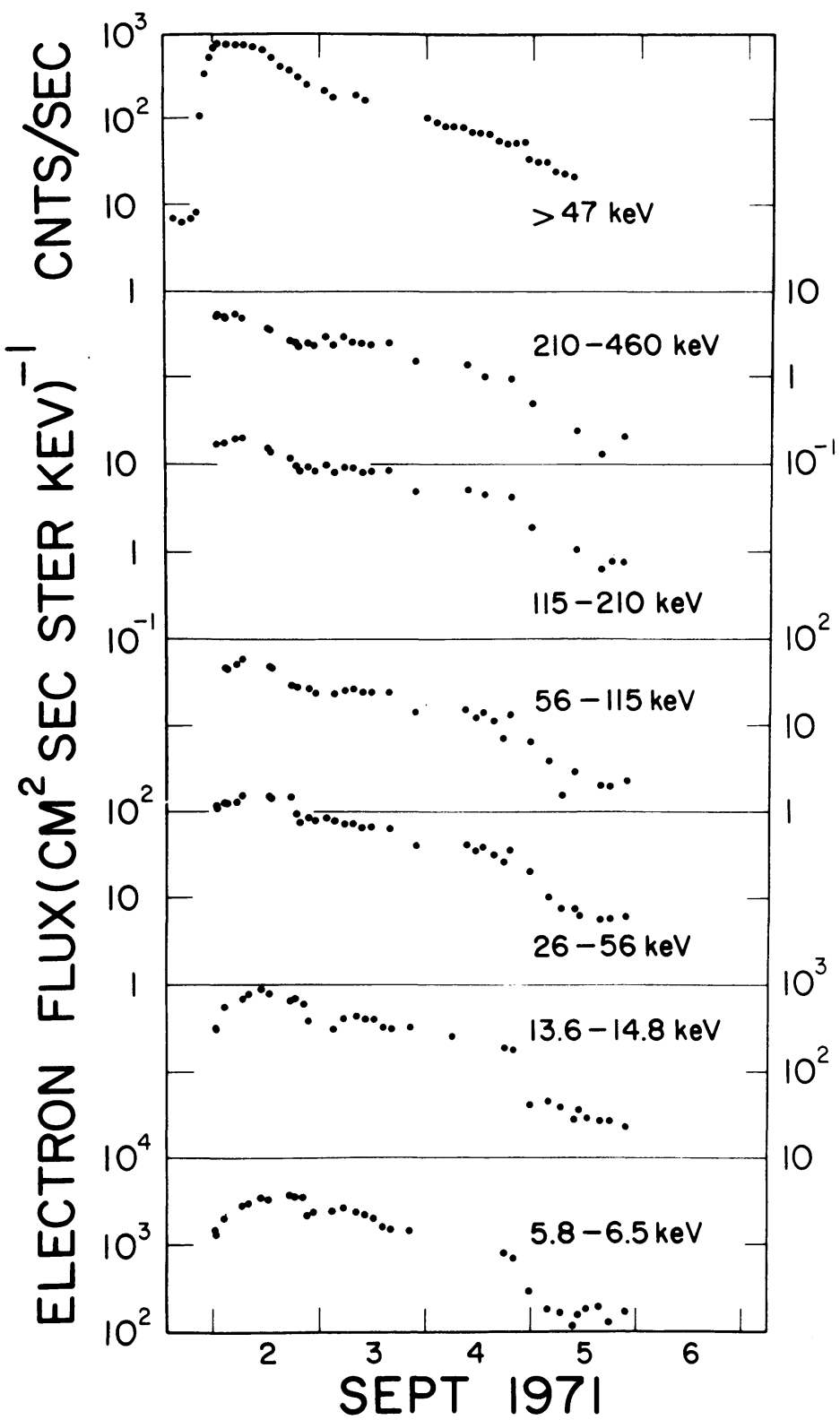

Fig. 3. The low energy electron observations for the flare event of 1 September 1971.

the peak in the particle density occurs at

$$
t_{\max }=\frac{r-r_{0}}{(\eta+1) k_{0}} .
$$

Taking $r \approx 1 \mathrm{AU}$ and $r_{0} \approx 7 \times 10^{5} \mathrm{~km}$ (one solar radius), we find that $\eta t_{\max } k_{0}>10^{13}$ 
$\mathrm{cm} \gg 2\left(r r_{0}\right)^{1 / 2} \approx 2 \times 10^{12} \mathrm{~cm}$ for $\mu>1$. From the time of maximum in each channel we have estimated $k_{0}$ for $V=400 \mathrm{~km} \mathrm{~s}^{-1}$.

The diffusion coefficients range from $\sim 1$ to $5 \times 10^{20} \mathrm{~cm}^{2} \mathrm{~s}^{-1}$ at $1 \mathrm{AU}$, comparable in value to those derived earlier by Lupton and Stone (1973) and others at energies of $\sim 1$ to $10 \mathrm{MeV}$. There is a discernible, although weak, trend to lower values of $k$ at lower energies. In this range of $k$ convection and adiabatic energy loss clearly play an important role in the particle propagation. Thus, it is likely that these particles were emitted at the Sun with substantially higher energies, and then lost energy through repeated scatterings with the magnetic field in the expanding solar wind. Estimates by Palmer (1973) from Monte-Carlo simulations indicate that for these values of $k$ typically $\gtrsim 75 \%$ of the original particle energy is lost by the time of maximum intensity at $1 \mathrm{AU}$.

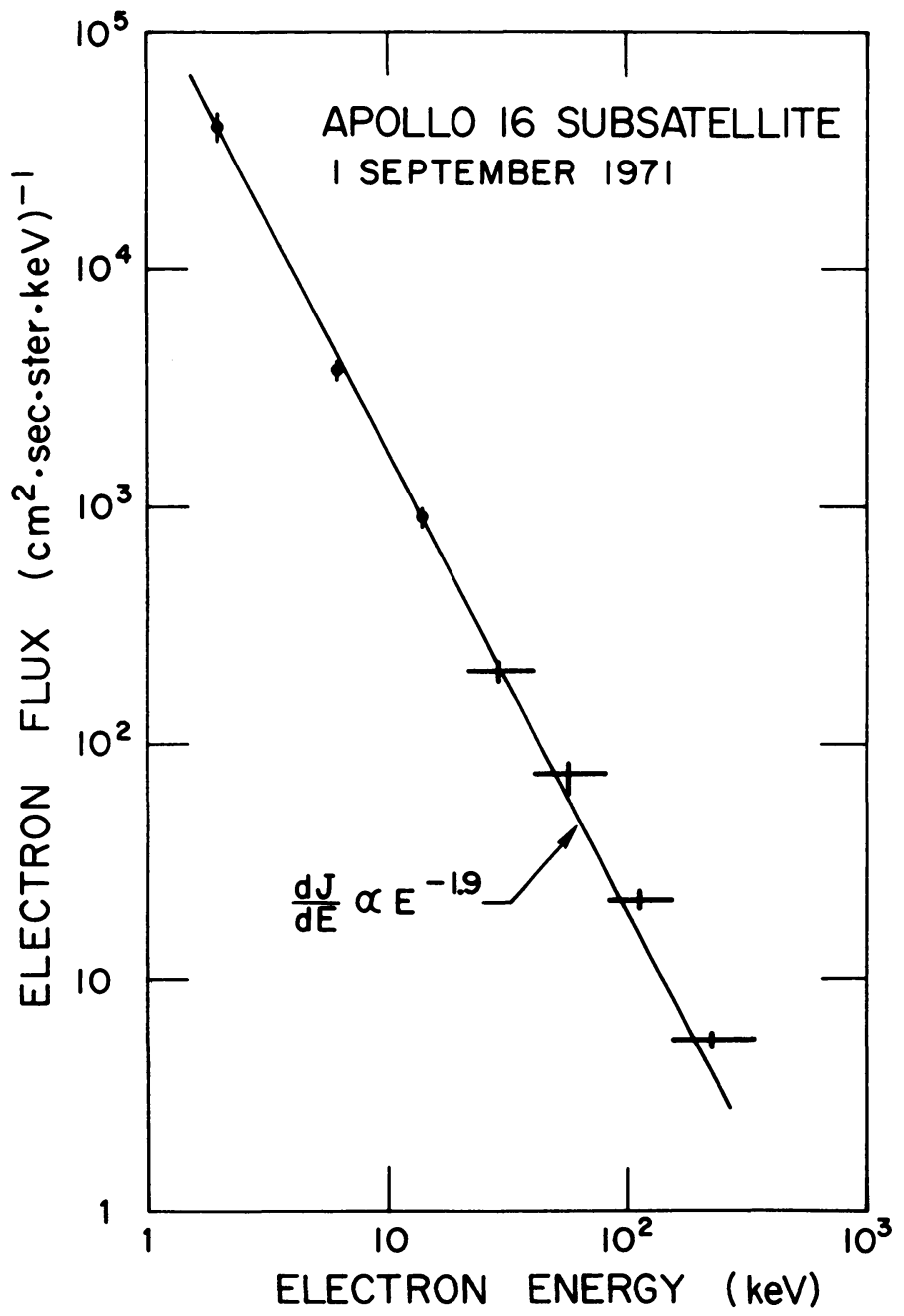

Fig. 4. The electron energy spectrum for the 1 September 1971 event. 
We can obtain a lower limit to the total energy in emitted protons by assuming, unrealistically, that no energy is lost to adiabatic deceleration in the propagation of these protons from the Sun to the Earth. To estimate the number of protons, we assume the protons fill an $\sim 100^{\circ}$ cone of interplanetary field lines uniformly out to $\sim 1.5$ AU by the time of maximum intensity at $1 \mathrm{AU}$. Then the lower limit on the total energy contained in protons above $\sim 0.04 \mathrm{MeV}$ is approximately

$$
\mathscr{E}_{\text {protons }}(E>0.04 \mathrm{MeV}) \gtrsim 5.4 \times 10^{28} \mathrm{erg}
$$

compared with about $2 \times 10^{28} \mathrm{erg}$ above $10 \mathrm{MeV}$. The actual total energy in protons emitted by the flare may be an order of magnitude or more higher than these numbers since the protons most likely started with several times the energy at the Sun that they were observed to have at $1 \mathrm{AU}$, and since protons below $0.04 \mathrm{MeV}$ were not included. The 1 September 1971 flare was not a large proton event at $1 \mathrm{AU}$; the August 4,1972 event was two to three orders of magnitude more intense. We note that previous estimates of the energy release in a large solar flare only took into account protons above $\sim 10 \mathrm{MeV}$ (Hundhausen, 1972) and gave a figure of $\sim 2 \times 10^{31} \mathrm{erg}$. If a similar proton energy spectrum exists for those events as in this event, then estimates of the total energy in energetic protons must be revised upward by a minimum factor of $\sim 3$, to $\gtrsim 6 \times 10^{31} \mathrm{erg}$. If the energy losses in propagation are taken into account energetic protons may easily contain a major portion of the total flare energy.

The $\mathrm{d} E / \mathrm{d} x$ energy loss in the solar atmosphere must be very small for the electrons since the spectrum extends as a smooth power law to $\sim 2 \mathrm{keV}$. We obtained an upper limit to the amount of material traversed of $\sim 5 \mu \mathrm{g} \mathrm{cm}^{-2}$ of ionized hydrogen (Lin, 1972). If we attribute the bending in the proton spectrum just to $\mathrm{d} E / \mathrm{d} x$ energy loss, then the amount of material traversed is $\sim 20 \mu \mathrm{g} \mathrm{cm}^{-2}$ of ionized hydrogen. These values are remarkably small and may indicate either that (1) the acceleration processes occur high in the solar corona or that (2) the energy loss formulae are inapplicable, as for example in a very hot plasma where the average particle energy, $\sim K T$, is on the order to tens of $\mathrm{keV}$ (Anderson, 1972).

\section{Summary}

The observations presented here show that protons of energies down to $\sim 0.4 \mathrm{MeV}$ at $1 \mathrm{AU}$ are impulsively emitted into interplanetary medium from solar flares. The energy contained in these low energy protons is at least $\sim 3$ times the energy in $>10 \mathrm{MeV}$ protons, and thus, these protons may constitute a major portion of the total flare energy in large proton flares.

\section{Acknowledgements}

This research was supported in part by NASA contract NAS 9-10509 and NASA grant NGL 05-003-017. 


\section{References}

Anderson, K. A.: 1972, Solar Phys. 27, 442.

Anderson, K. A., Chase, L. M., Lin, R. P., McCoy, J. E., and McGuire, R. E. : 1972, J. Geophys. Res. 77, 4611.

Fisk, L. A. and Axford, W. I.: 1968, J. Geophys. Res. 73, 4396.

Hundhausen, A. J.: 1972, in C. P. Sonett, P. J. Coleman, Jr., and J. M. Wilcox (eds.), Solar Wind, NASA SP-308, p. 393.

Jokipii, J. R. : 1971, Phys. Rev. Letters 26, 666.

Kane, S. R.: 1974, this volume, p. 105.

Lin, R. P.: 1972, in R. Ramaty and R. G. Stone (eds.), Proc. Symposium on High Energy Phenomena on the Sun, Goddard Space Flight Center, Greenbelt, Maryland, Sept. 28-30.

Lin, R. P.: 1974, Space Sci. Rev. 16, 189.

Lin, R. P. and Hudson, H. S.: 1971, Solar Phys. 17, 412.

Lupton, J. E. and Stone, E. C.: 1973, J. Geophys. Res. 78, 1007.

Murray, S. S., Stone, E. C., and Vogt, R. E.: 1971, Phys. Rev. Letters 26, 663.

Ng, C. K. and Gleeson, L. J.: 1971, Solar Phys. 20, 166.

Palmer, I. D.: 1973, Solar Phys. 30, 235.

Parker, E. N. : 1965, Planetary Space Sci. 13, 9.

Verzariu, P. and Krimigis, S. M.: 1972, J. Geophys. Res. 77, 3985.

\section{DISCUSSION}

Newkirk: Could you put a number on the amount of material which has been encountered by your low energy protons?

Lin: $15 \mu \mathrm{g} \mathrm{cm}^{-2}$ if there is no energy loss.

Dryer: It is very interesting to see your estimate of energy for greater than $50 \mathrm{keV}$ protons (for the 1972 August 4 flare) as $5 \times 10^{32} \mathrm{erg}$. The hourly-averaged bulk velocities for Pioneer 9 show about $100 \mathrm{~km} \mathrm{~s}^{-1}$ for the lower energy protons. Although additional detailed study of the finer time-resolved data is forthcoming, I could hazard a guess at this time that - on the basis of estimates for flares in the past - the energy would be at least equal to the amount you presented for $>50 \mathrm{keV}$ protons.

Lin: The estimated energy continues to rise. 